Research Article

\title{
On the permanental polynomial and permanental sum of signed graphs
}

\author{
Zikai Tang, Qiyue Li, Hanyuan Deng* \\ MOE-LCSM, School of Mathematics and Statistics, Hunan Normal University, Changsha, Hunan, P. R. China
}

(Received: 4 January 2022. Received in revised form: 16 February 2022. Accepted: 21 February 2022. Published online: 24 February 2022.)

(C) 2022 the authors. This is an open access article under the CC BY (International 4.0) license (www.creativecommons.org/licenses/by/4.0/).

\begin{abstract}
Let $\dot{G}=(G, \sigma)$ be a signed graph, where $G$ is its underlying graph and $\sigma$ is its sign function (defined on the edge set $E(G)$ of $G)$. Let $A(\dot{G})$ be the adjacency matrix of $\dot{G}$. The polynomial $\pi(\dot{G}, x)=\operatorname{per}(x I-A(\dot{G}))$ is called the permanental polynomial of $\dot{G}$, where $I$ is the identity matrix and per denotes the permanent of a matrix. In this paper, we obtain the coefficients of the permanental polynomial of a signed graph in terms of its structure. We also establish the recursion formulas for the permanental polynomial of a signed graph. Moreover, we investigate the permanental sum $P S(\dot{G})$ of a signed graph $\dot{G}$, give the recursion formulas for the permanental sum $P S(\dot{G})$, and show that the equation $P S(\dot{G})=P S(G)$ holds for trees and unicyclic graphs, where $P S(G)$ is the permanental sum of the underlying graph $G$ of $\dot{G}$.
\end{abstract}

Keywords: adjacency matrix; permanental polynomial; coefficient; recursion formulas; permanental sum.

2020 Mathematics Subject Classification: 05C31, 05C75.

\section{Introduction}

The permanent of an $n \times n$ real matrix $A=\left(a_{i j}\right)$, with $i, j \in\{1,2, \ldots, n\}$, is defined as

$$
\operatorname{per}(A)=\sum_{\tau} \Pi_{i=1}^{n} a_{i \tau(i)}
$$

where the sum is taken over all permutations $\tau$ of $\{1,2, \ldots, n\}$. Let $A(G)$ be the adjacency matrix of a graph $G=(V, E)$ with $n$ vertices. The polynomial

$$
\Phi(G, x)=\operatorname{det}(x I-A(G))=\sum_{k=0}^{n} a_{k} x^{n-k},
$$

is called the characteristic polynomial of $G$, where $I$ is the $n \times n$ identity matrix. The polynomial

$$
\pi(G, x)=\operatorname{per}(x I-A(G))=\sum_{k=0}^{n} b_{k} x^{n-k},
$$

is called the permanental polynomial of $G$ [7]. The characteristic polynomial and the permanental polynomial are important among the well-studied graph polynomials. Valiant [9] has shown that computing the permanent of matrices is $\sharp$ P-complete even when restricted to $(0,1)$-matrices. It is difficult to compute the permanental polynomials of graphs. Numerous works were done on the adjacency permanental polynomials of graphs, including the relations between the adjacency permanental and characteristic polynomials of graphs, the coefficients and roots of the adjacency permanental polynomial of a graph [1-3,16-18]. It was shown that the coefficients of the characteristic and permanental polynomials of graphs are related to graphs' structures [3, 4,7].

A linear graph (or a Sachs graph) is a graph in which each component is a single edge or a cycle. A linear subgraph of a graph $G$ is termed as a subgraph whose components are cycles or single edges. A linear subgraph with $k$ vertices is denoted by $U_{k}$. Then

$$
a_{k}=\sum_{U_{k} \subseteq G}(-1)^{p\left(U_{k}\right)} 2^{c\left(U_{k}\right)}(1 \leq k \leq n),
$$

and

$$
b_{k}=(-1)^{k} \sum_{U_{k} \subseteq G} 2^{c\left(U_{k}\right)}(1 \leq k \leq n),
$$

where the summations range over all linear subgraphs $U_{k}$ of $G, p\left(U_{k}\right)$ is the number of components of $U_{k}$ and $c\left(U_{k}\right)$ is the 
number of cycles of $U_{k}$. For a bipartite graph $G, b_{n}$ is equal to the square of the number $m(G)$ of perfect matchings of $G$ [6], i.e.

$$
b_{n}=m^{2}(G) \text {. }
$$

Let $\sigma: E(G) \rightarrow\{+,-\}$ be a mapping defined on the edge set of $G$ and $\dot{G}=(G, \sigma)$ a signed graph, where $G$ is its underlying graph and $\sigma$ is its sign function (or signature). Hence, signed graphs are sometimes treated as weighted graphs, whose (edge) weights belong to $\{1,-1\}$. An edge $e$ is positive (negative) if $\sigma(e)=+$ (resp. $\sigma(e)=-$ ). If all edges in $\dot{G}$ are positive (negative), then $\dot{G}$ is denoted by $(G,+)$ (resp. $(G,-)$ ). A cycle of $\dot{G}$ is said to be balanced (or positive) if it contains an even number of negative edges, otherwise it is unbalanced (or, negative). A signed graph is said to be balanced if all its cycles are balanced; otherwise, it is unbalanced.

Let $\dot{G}$ be a signed graph on $n$ vertices. The adjacency matrix of $\dot{G}$ is $A(\dot{G})=\left(a_{i j}^{\sigma}\right)$ with $a_{i j}^{\sigma}=\sigma\left(v_{i} v_{j}\right) a_{i j}$, where $\left(a_{i j}\right)$ is the adjacency matrix of the underlying graph $G=(V, E)$ with $V=\left\{v_{1}, v_{2}, \ldots, v_{n}\right\}$. Then

$$
\Phi(\dot{G}, x)=\operatorname{det}(x I-A(\dot{G}))=\sum_{k=0}^{n} c_{k} x^{n-k}
$$

is the characteristic polynomial [5] of $\dot{G}, c_{0}=1$ and

$$
c_{k}=\sum_{U_{k} \subseteq \dot{G}}(-1)^{p\left(U_{k}\right)+s\left(U_{k}\right)} 2^{c\left(U_{k}\right)}=\sum_{U_{k} \subseteq \dot{G}}(-1)^{p\left(U_{k}\right)+c^{-}\left(U_{k}\right)} 2^{c\left(U_{k}\right)}(1 \leq k \leq n),
$$

for any $k(1 \leq k \leq n)$, where $s\left(U_{k}\right)$ is the number of negative edges in cycles of $U_{k}, p\left(U_{k}\right)$ is the number of components of $U_{k}$, $c\left(U_{k}\right)$ is the number of cycles of $U_{k}$ and $c^{-}\left(U_{k}\right)$ is the number of negative cycles of $U_{k}$.

Similarly, we introduce the permanental polynomial of signed graph $\dot{G}$ defined as

$$
\pi(\dot{G}, x)=\operatorname{per}(x I-A(\dot{G}))=\sum_{k=0}^{n} s_{k} x^{n-k}
$$

and its permanental sum $P S(\dot{G})$ as

$$
P S(\dot{G})=\sum_{k=0}^{n}\left|s_{k}\right|
$$

In this paper, we investigate the permanental polynomial of a signed graph and obtain its coefficients in terms of the graph structure, and establish the recursion formulas for the permanental polynomial and the permanental sum $P S(\dot{G})$ of signed graph $\dot{G}$, and show that $P S(\dot{G})=P S(G)$ for trees and unicyclic graphs, where $P S(G)$ is the permanental sum of its underlying graph $G$ introduced in [12].

\section{The coefficients of the permanental polynomial of a signed graph}

In this section, we give a graphical interpretation of the coefficients of the permanental polynomial of a signed graph using its linear subgraphs.

Let $A$ be an $m \times n$ matrix. If $S \subseteq\{1, \ldots, m\}$ and $T \subseteq\{1, \ldots, n\}$, then $A[S \mid T]$ denotes the submatrix of $A$ determined by the rows corresponding to $S$ and the columns corresponding to $T$.

Lemma 2.1 (see [8]). If $A$ is an $n \times n$ matrix and $\operatorname{per}(x I-A)=\sum_{k=0}^{n} b_{k} x^{n-k}$, then $b_{0}=1$ and $b_{k}=(-1)^{k} \sum_{|T|=k} \operatorname{per}(A[T \mid T])$ for $1 \leq k \leq n$.

Theorem 2.1. Let $\dot{G}$ be a signed graph with $n$ vertices and $\pi(\dot{G}, x)=\sum_{k=1}^{n} s_{k} x^{n-k}$ be its permanental polynomial. Then $s_{0}=1$ and

$$
s_{k}=\sum_{U_{k} \subseteq \dot{G}}(-1)^{k+c^{-}\left(U_{k}\right)} 2^{c\left(U_{k}\right)}
$$

for $1 \leq k \leq n$, where $c^{-}\left(U_{k}\right)$ is the number of negative cycles of $U_{k}$ and $c\left(U_{k}\right)$ is the number of cycles of $U_{k}$.

Proof. Let $\dot{A}=A(\dot{G})$ be the adjacency matrix of $\dot{G}$. Clearly, $s_{0}=1$. By Lemma 2.1, we have $s_{k}=(-1)^{k} \sum_{|T|=k} \operatorname{per}(\dot{A}[T \mid T])$ for $1 \leq k \leq n$. We first prove that

$$
s_{n}=(-1)^{n} \operatorname{per}(\dot{A})=\sum_{U_{n} \subseteq \dot{G}}(-1)^{n+c^{-}\left(U_{n}\right)} 2^{c\left(U_{n}\right)} .
$$

Let $\pi=\pi_{1} \pi_{2} \ldots \pi_{n}$ be a permutation of $\{1,2, \ldots, n\}$. Then $\operatorname{per}(\dot{A})=\sum_{\pi} \Pi_{i=1}^{n} a_{i, \pi_{i}}$, where the sum is taken over all permutations $\pi$ of $\{1,2, \ldots, n\}$. The term vanishes if $a_{i, \pi_{i}}=0$, i.e., $\left\{v_{i}, v_{\pi_{i}}\right\}$ is not an edge of $\dot{G}$ for some $i \in\{1,2, \ldots, n\}$. 
Thus, if the term corresponding to a permutation $\pi$ is non-zero, then $\pi$ can be expressed uniquely as the composition of disjoint cycles or some $K_{2}$. Each $K_{2}$ corresponds to the factors $a_{i, j} a_{j, i}$, which in turn signifies a single edge $v_{i} v_{j}$ in $\dot{G}$. Each cycle $(p q r \ldots t)$ of length greater than two corresponds to the factors $a_{p q} a_{q r} \ldots a_{t p}$, and signifies a simple circuit $v_{p}, v_{q}, \ldots, v_{t}$ in $\dot{G}$. Consequently, each non-vanishing term in the permanent expansion gives rise to a linear subgraph $U_{n}$ of $G$ with $V(S)=V(G)$. The number of such $\pi^{\text {'s }}$ - arising from a given $U_{n}$ is $2^{c\left(U_{n}\right)}$, since there are two ways of choosing the corresponding cycle in $\pi$ for each circuit in $U_{n}$. Thus, each $U_{n}$ contributes $(-1)^{c^{-}\left(U_{n}\right)} 2^{c\left(U_{n}\right)}$ to the permanent of $\dot{A}$, and we have that

$$
s_{n}=(-1)^{n} \operatorname{per}(\dot{A})=\sum_{U_{n} \subseteq \dot{G}}(-1)^{n+c^{-}\left(U_{n}\right)} 2^{c\left(U_{n}\right)} .
$$

Similarly, we can deduce

$$
s_{k}=\sum_{U_{k} \subseteq \dot{G}}(-1)^{k+c^{-}\left(U_{k}\right)} 2^{c\left(U_{k}\right)}
$$

for $1 \leq k \leq n-1$.

Theorem 2.2. For a graph $G$ on $n$ vertices, there is a signed graph $\dot{G}$ of $G$ with $\left|s_{k}\right|=\left|b_{k}\right|$ if and only if the number of negative cycles in any linear subgraph with $k$ vertices has the same parity.

Proof. First, $b_{k}=0$ if and only if no linear subgraph $U_{k}$ with $k$ vertices exists in $G$, which is the case if and only if no linear subgraph $U_{k}$ with $k$ vertices exists in $\dot{G}$. Thus, it is trivial for the case $b_{k}=0$. Next, we consider the case $b_{k}>0$.

If the number of negative cycles in any linear subgraph $U_{k}$ with $k$ vertices has the same parity, then $(-1)^{c^{-}\left(U_{k}\right)}=1$ (resp. -1) for all $U_{k}$. So, we have

$$
\left|s_{k}\right|=\left|\sum_{U_{k} \subseteq \dot{G}}(-1)^{k+c^{-}\left(U_{k}\right)} 2^{c\left(U_{k}\right)}\right|=\sum_{U_{k} \subseteq \dot{G}} 2^{c\left(U_{k}\right)}=\sum_{U_{k} \subseteq G} 2^{c\left(U_{k}\right)}
$$

and

$$
\left|b_{k}\right|=\left|(-1)^{k} \sum_{U_{k} \subseteq G} 2^{c\left(U_{k}\right)}\right|=\sum_{U_{k} \subseteq G} 2^{c\left(U_{k}\right)} .
$$

So we have $\left|s_{k}\right|=\left|b_{k}\right|$.

On the other hand, if $\left|s_{k}\right|=\left|b_{k}\right|$, then all the linear subgraphs $U_{k}$ satisfy

$$
\sum_{U_{k} \subseteq G} 2^{c\left(U_{k}\right)}=\sum_{U_{k} \subseteq \dot{G}} 2^{c\left(U_{k}\right)}=\sum_{U_{k} \subseteq \dot{G}}(-1)^{c^{-}\left(U_{k}\right)} 2^{c\left(U_{k}\right)}
$$

or

$$
\sum_{U_{k} \subseteq G} 2^{c\left(U_{k}\right)}=\sum_{U_{k} \subseteq \dot{G}} 2^{c\left(U_{k}\right)}=-\sum_{U_{k} \subseteq \dot{G}}(-1)^{c^{-}\left(U_{k}\right)} 2^{c\left(U_{k}\right)} .
$$

This means that the number of negative cycles in any linear subgraph $U_{k}$ with $k$ vertices has the same parity.

By the definition of balanced signed graph and Theorem 2.2, we can get the following results.

Corollary 2.1. Let a graph $G$ on $n$ vertices and its signed graph $\dot{G}$ is a balanced signed graph. Then $s_{k}=b_{k}, 1 \leq k \leq n$.

Corollary 2.2. Let $\dot{T}$ be any signed graph of a tree $T$. Then $\operatorname{per}(x I-A(\dot{T}))=\operatorname{per}(x I-A(T))$.

Let $G$ be a bipartite graph with $n$ vertices. We know $b_{n}=m^{2}(G)$ in [6]. From Theorem 2.2, we can obtain

Corollary 2.3. For a bipartite graph $G$ on $n$ vertices, there is a signed graph $\dot{G}$ of $G$ with $\left|s_{n}\right|=m^{2}(G)$ if and only if the number of negative cycles in any linear subgraph with nertices has same parity.

Corollary 2.4. There is a signed graph $\dot{G}$ of the bipartite perfect matching $n$ order graph $G$ with $s_{n}=m^{2}(G)$ if and only if the number of negative cycles in any the linear subgraph with $n$ vertices is even.

Proof. Let $n=2 p$. Since $G$ has a perfect matching, $p K_{2}$ is a linear subgraph $U_{n}$ of $\dot{G}$ and the number of negative cycles of $p K_{2}$ is zero. By Corollary 2.3, the result holds.

We know that the perfect matching of a tree is unique if it exists.

Corollary 2.5. Any signed tree $\dot{T}$ of the perfect matching $n$ order tree $T$ with $s_{n}=\operatorname{per}(A(\dot{T}))=\operatorname{per}(A(T))=1$. 


\section{Recursion formulas for the permanental polynomial of a signed graph}

The relationships between the permanental polynomial of a signed graph and the ones of its subgraphs are discussed in this section. Let $G$ be a graph with a vertex subset $S \subseteq V(G)$. Denote by $G-S$ the graph obtained by deleting all the vertices in $S$ from $G$ together with all edges incident with $S$. In particular, if $S=\{v\}$ with $v \in V(G)$, we write $G-\{v\}$ simply by $G-v$.

Theorem 3.1. Let $e=(u, v)$ be an edge of a signed graph $\dot{G}$ and $\mathcal{C}_{e}(\dot{G})$ the set of cycles in $\dot{G}$ containing $e$. Then

$$
\pi(\dot{G}, x)=\pi(\dot{G}-e, x)+\pi(\dot{G}-u-v, x)+2 \Sigma_{C \in \mathcal{C}_{e}(\dot{G})}(-1)^{|V(C)|+r(C)} \pi(\dot{G}-V(C), x),
$$

where $r(C)=1$ when $C$ is negative in $\dot{G}$, and $r(C)=0$ when $C$ is positive.

Proof. Let $\pi(\dot{G}, x)=\sum_{k=0}^{n} s_{k} x^{n-k}$. By Theorem 2.1, the coefficient $s_{k}$ can be expressed in terms of the linear subgraphs of $\dot{G}$. We show that if a linear subgraph $U_{k}$ contributes to $s_{k}$ on the left side of (9), then there is a linear subgraph that contributes a corresponding amount to one of the terms on the right. Suppose that $U_{k}$ contributes $m$ to the coefficient $s_{k}$ of $x^{n-k}$ on the left. We consider the following cases of $U_{k}$.

Case 1: $e \notin U_{k}$. Let $W_{k}=U_{k}$, then $W_{k}$ is a linear subgraph of $\dot{G}-e$ and it contributes $m$ to the coefficient of $x^{n-k}$ in $\pi(\dot{G}-e, x)$.

Case 2: $e=u v$ is a component of $U_{k}$. Let $W_{k-2}=U_{k}-u-v$, then $W_{k-2}$ is a linear subgraph of $\dot{G}-u-v$. By (8), $W_{k-2}$ contributes

$$
(-1)^{k-2+c^{-}\left(W_{k-2}\right)} 2^{c\left(W_{k-2}\right)}=(-1)^{k+c^{-}\left(U_{k}\right)} 2^{c\left(U_{k}\right)}=m
$$

to the coefficient of $x^{n-k}$ in $\pi(\dot{G}-u-v, x)$.

Case 3: $e=u v$ belongs to a cycle $C$ of $U_{k}$. Then $W^{e}=U_{k}-V(C)$ is a linear subgraph of $\dot{G}-V(C)$. In this case, $W^{e}$ contributes

$$
(-1)^{k-|V(C)|+c^{-}\left(W^{e}\right)} 2^{c\left(W^{e}\right)}=(-1)^{k-|V(C)|+c^{-}\left(W^{e}\right)} 2^{c\left(U_{k}\right)-1}
$$

to the coefficient of $x^{n-k}$ in $\pi(\dot{G}-V(C), x)$. If $C$ is negative in $\dot{G}$, then

$$
(-1)^{k-|V(C)|+c^{-}\left(W^{e}\right)} 2^{c\left(W^{e}\right)}=(-1)^{k-|V(C)|+c^{-}\left(U_{k}\right)-1} 2^{c\left(U_{k}\right)-1}=\frac{1}{2}(-1)^{|V(C)|+1} m .
$$

If $C$ is positive in $\dot{G}$, then

$$
(-1)^{k-|V(C)|+c^{-}\left(W^{e}\right)} 2^{c\left(W^{e}\right)}=(-1)^{k-|V(C)|+c^{-}\left(U_{k}\right)} 2^{c\left(U_{k}\right)-1}=\frac{1}{2}(-1)^{|V(C)|} m .
$$

Thus, $W^{e}$ contributes $m$ to the coefficient of $x^{n-k}$ in $2(-1)^{|V(C)|+r(C)} \pi(\dot{G}-V(C), x)$, where $r(C)=1$ when $C$ is negative in $\dot{G}$, and $r(C)=0$ when $C$ is positive.

By all the above cases, the result holds.

Similarly, we can get the following result.

Theorem 3.2. Let $v$ be a vertex of a signed graph $\dot{G}$ and $\mathcal{C}_{v}(\dot{G})$ the set of cycles in $\dot{G}$ containing $v$. Then

$$
\pi(\dot{G}, x)=x \pi(\dot{G}-v, x)+\Sigma_{u \sim v} \pi(\dot{G}-u-v, x)+2 \Sigma_{C \in \mathcal{C}_{v}(\dot{G})}(-1)^{|V(C)|+r(C)} \pi(\dot{G}-V(C), x),
$$

where $r(C)=1$ when $C$ is negative in $\dot{G}$, and $r(C)=0$ when $C$ is positive.

\section{Permanental sum $P S(\dot{G})$ of a signed graph}

Some works were done on the permanental sum of graphs [10-15]. The permanental sum $P S(\dot{G})$ of signed graphs is discussed in this section. By definition of the permanental sum $P S(\dot{G})$ of a signed graph $\dot{G}$ and Theorem 2.1, we have

$$
P S(\dot{G})=\sum_{k=0}^{n}\left|s_{k}\right|=\sum_{k=0}^{n}\left|\sum_{U_{k} \subseteq \dot{G}}(-1)^{k+c^{-}\left(U_{k}\right)} 2^{c\left(U_{k}\right)}\right|=\sum_{k=0}^{n}\left|\sum_{U_{k} \subseteq \dot{G}}(-1)^{c^{-}\left(U_{k}\right)} 2^{c\left(U_{k}\right)}\right|,
$$

and

$$
P S(\dot{G})=\operatorname{per}(I+A(\dot{G}))
$$

By Theorem 2.2 and definition of the permanental sum $P S(\dot{G})$ of a signed graph $\dot{G}$, we have the next result. 
Theorem 4.1. Let $\dot{G}$ be a signed graph. If the number of negative cycles in any linear subgraph $U_{k}$ with $k$ vertices has same parity for $1 \leq k \leq n$, then $P S(\dot{G})=P S(G)$.

In [12], Wu and Lai showed that the permanental sum of a graph satisfies the following identities.

Lemma 4.1 (see [12]). (i) Let $G$ and $H$ be two vertex disjoint connected graphs. Then

$$
P S(G \cup H)=P S(G) P S(H) .
$$

(ii) Let $e=u v$ be an edge of graph $G$. Then

$$
P S(G)=P S(G-e)+P S(G-v-u)+2 \sum_{C \in \mathcal{C}_{e}(G)} P S(G-V(C)) .
$$

(iii) Let $v$ be a vertex of graph G. Then

$$
P S(G)=P S(G-v)+\sum_{u \in N_{G}(v)} P S(G-v-u)+2 \sum_{C \in \mathcal{C}_{v}(G)} P S(G-V(C)) .
$$

Next, we will consider that the recursion formulas for the permanental sum $P S(\dot{G})$ of signed graph $\dot{G}$.

Theorem 4.2. (i) Let $\dot{G}$ and $\dot{H}$ be two vertex disjoint connected balanced signed graphs. Then

$$
P S(\dot{G} \cup \dot{H})=P S(\dot{G}) P S(\dot{H}) .
$$

(ii) Let $e=u v$ be an edge of signed graph $\dot{G}$. Then

$$
P S(\dot{G})=P S(\dot{G}-e)+P S(\dot{G}-v-u)+2 \sum_{\left.C \in \mathcal{C}_{e}(\dot{G})\right)}(-1)^{r(C)} P S(\dot{G}-V(C)) .
$$

(iii) Let $v$ be a vertex of signed graph $\dot{G}$. Then

$$
P S(\dot{G})=P S(\dot{G}-v)+\sum_{u \in N_{\dot{G}}(v)} P S(\dot{G}-v-u)+2 \sum_{C \in \mathcal{C}_{v}(\dot{G})}(-1)^{r(C)} P S(\dot{G}-V(C)) .
$$

where $r(C)=1$ when $C$ is negative in $\dot{G}$, and $r(C)=0$ when $C$ is positive.

Proof. (i) Each linear subgraph with $k$ vertices in $\dot{G} \cup \dot{H}$ consists of a linear subgraph with $t$ in $\dot{G}$ together with a linear subgraph with $k-t$ vertices in $\dot{H}$, where $0 \leq t \leq k$. Since $\dot{G}$ and $\dot{H}$ are connected balanced signed graphs, by Theorem 2.1, we have

$$
P S(\dot{G} \cup \dot{H})=\sum_{k=0}^{n}\left|s_{k}(\dot{G} \cup \dot{H})\right|=\sum_{k=0}^{n} \sum_{t=0}^{k}\left|s_{t}(\dot{G}) s_{k-t}(\dot{H})\right|=P S(\dot{G}) P S(\dot{H}) .
$$

Now, let $L_{i}$ denote the collection of all linear subgraphs of $\dot{G}$ with $i$ vertices.

(ii) Let $e=u v \in E(G)$ be a given edge, and $L_{i}^{\prime}(G, e)=\left\{H \in L_{i}: e \in E(H)\right\}$ and $L_{i}^{\prime \prime}(G, e)=\left\{H \in L_{i}: e \notin E(H)\right\}$. Then, $\left|L_{i}^{\prime \prime}(G, e)\right|=\left|s_{i}(G-u v)\right|$. For each $H \in L_{i}^{\prime}(G, e)$, either $e=u v$ itself is a component of $H$, or $e$ lies in a cycle of $H$. It follows that $\mid\left\{H \in L_{i}^{\prime}(G, e): e\right.$ is a component of $\left.H\right\}|=| s_{i-2}(G-u-v) \mid$, and

$$
\mid\left\{H \in L_{i}^{\prime}(G, e): e \text { lies in a cycle of } H\right\}\left|=2 \sum_{k=0}^{i}\right| \sum_{\left.C_{k} \in \mathcal{C}_{e}(\dot{G})\right)}(-1)^{r\left(C_{k}\right)} s_{i-k}\left(G-V\left(C_{k}\right)\right) \mid .
$$

Thus,

$$
\left|s_{i}(\dot{G})\right|=\left|s_{i}(\dot{G}-u v)\right|+\left|s_{i-2}(\dot{G}-u-v)\right|+2 \sum_{k=0}^{i}\left|\sum_{\left.C_{k} \in \mathcal{C}_{e}(\dot{G})\right)}(-1)^{r\left(C_{k}\right)} s_{i-k}\left(\dot{G}-V\left(C_{k}\right)\right)\right| .
$$

for all positive integers $i$. It follows that

$$
\begin{aligned}
P S(\dot{G}) & =\sum_{k=0}^{n}\left|s_{i}(\dot{G})\right| \\
& =\sum_{k=0}^{n}\left(\left|s_{i}(\dot{G}-u v)\right|+\left|s_{i-2}(\dot{G}-u-v)\right|+2 \sum_{k=0}^{i}\left|\sum_{\left.C_{k} \in \mathcal{C}_{e}(\dot{G})\right)}(-1)^{r\left(C_{k}\right)} s_{i-k}\left(\dot{G}-V\left(C_{k}\right)\right)\right|\right)
\end{aligned}
$$




$$
=P S(\dot{G}-e)+P S(\dot{G}-v-u)+2 \sum_{\left.C \in \mathcal{C}_{e}(\dot{G})\right)}(-1)^{r(C)} P S(\dot{G}-V(C)) .
$$

(iii) Let $v \in V(G)$ be a given vertex, and $L_{i}^{\prime}(G, v)=\left\{H \in L_{i}: v \in E(H)\right\}$ and $L_{i}^{\prime \prime}(G, v)=\left\{H \in L_{i}: v \notin V(H)\right\}$. Then, $\left|L_{i}^{\prime \prime}(G, v)\right|=\left|s_{i}(G-v)\right|$. For each $H \in L_{i}(G, v)$, either $v$ is an endpoint of some single, or $v$ lies in a cycle of $H$. It follows that $\mid\left\{H \in L_{i}^{\prime}(G, v): v\right.$ is an endpoint of some single $e=u v$ of $\left.H\right\}|=| s_{i-2}(G-u-v) \mid$, and

$$
\mid\left\{H \in L_{i}^{\prime}(G, v): v \text { lies in a cycle of } H\right\}\left|=2 \sum_{k=0}^{i}\right| \sum_{\left.C_{k} \in \mathcal{C}_{v}(\dot{G})\right)}(-1)^{r\left(C_{k}\right)} s_{i-k}\left(G-V\left(C_{k}\right)\right) \mid .
$$

Thus,

$$
\left|s_{i}(\dot{G})\right|=\left|s_{i}(\dot{G}-v)\right|+\left|s_{i-2}(\dot{G}-u-v)\right|+2 \sum_{k=0}^{i}\left|\sum_{\left.C_{k} \in \mathcal{C}_{v}(\dot{G})\right)}(-1)^{r\left(C_{k}\right)} s_{i-k}\left(\dot{G}-V\left(C_{k}\right)\right)\right| .
$$

Substituting this into the definition of $P S(\dot{G})$ yields

$$
P S(\dot{G})=P S(\dot{G}-v)+\sum_{u \in N_{\dot{G}}(v)} P S(\dot{G}-v-u)+2 \sum_{C \in \mathcal{C}_{v}(\dot{G})}(-1)^{r(C)} P S(\dot{G}-V(C)) .
$$

From Theorem 4.2 the next two corollaries immediately follows.

Corollary 4.1. (i) Let $e=u v$ be an edge of signed graph $\dot{G}$. If all cycles containing e are positive (or negative), then

$$
P S(\dot{G})=P S(\dot{G}-e)+P S(\dot{G}-v-u)+2 \sum_{\left.C \in \mathcal{C}_{e}(\dot{G})\right)} P S(\dot{G}-V(C)) .
$$

(ii) Let $v$ be a vertex of signed graph $\dot{G}$. If all cycles containing $v$ are positive (or negative), then

$$
P S(\dot{G})=P S(\dot{G}-v)+\sum_{u \in N_{\dot{G}(v)}} P S(\dot{G}-v-u)+2 \sum_{C \in \mathcal{C}_{v}(\dot{G})} P S(\dot{G}-V(C)) .
$$

Corollary 4.2. (i) Let $\dot{G}$ be a signed graph and e an edge of $\dot{G}$. Then $P S(\dot{G}-e)<P S(\dot{G})$.

(ii) Among all signed graphs with $n$ vertices, the graph $n K_{1}$ and the balance complete signed graph $K_{n}$ have, respectively, minimum and maximum permanental sum.

We know $P S\left(K_{2}\right)=P S\left(\dot{K}_{2}\right)=2$. From Theorem 4.2 the next result immediately follows.

Corollary 4.3. If $G$ is a tree or a unicyclic graph, then $P S(G)=P S(\dot{G})$ for any signed graph $\dot{G}$ of $G$.

Wu and Lai [12] determine the largest and smallest permanental sums among unicyclic graphs (trees). Denote by $F(n)$ the $n^{\text {th }}$ Fibonacci number.

Lemma 4.2 (see [12]). (i) Let $G$ be a tree with $n$ vertex, then

$$
n \leq P S(G) \leq F(n+1),
$$

where the left equality holds if and only if $T=S_{n}$, and the right equality holds if and only if $T=P_{n}$.

(ii) Let $G$ be a unicyclic graph with $n$ vertex, then

$$
2 n \leq P S(G) \leq 6 F(n-2)+2 F(n-3),
$$

where the left equality holds if and only if $T=S_{n}^{+}$(the graph obtained by adding a new edge to the star $S_{n}$ ), and the right equality holds if and only if $T=D_{3, n-3}$ (the graph obtained from the disjoint union of a cycle $C_{3}$ and a path $P_{n-3}$ by identifying one end of $P_{n-3}$ with one of the vertices of $C_{3}$ ).

By Corollary 4.3 and Lemma 4.2, we have

Corollary 4.4. (i) Let $G$ be a tree with $n$ vertex and $\dot{G}$ a signed graph of $G$, then

$$
n \leq P S(\dot{G}) \leq F(n+1),
$$

where the left equality holds if and only if $G=S_{n}$, and the right equality holds if and only if $G=P_{n}$.

(ii) Let $G$ be a unicyclic graph with $n$ vertex and $\dot{G}$ a signed graph of $G$, then

$$
2 n \leq P S(\dot{G}) \leq 6 F(n-2)+2 F(n-3),
$$

where the left equality holds if and only if $G=S_{n}^{+}$, and the right equality holds if and only if $G=D_{3, n-3}$. 


\section{Acknowledgement}

This work is supported by the Hunan Provincial Natural Science Foundation of China (through Grant No. 2020JJ4423) and the Department of Education of Hunan Province (through Grant No. 19A318). Valuable comments and suggestions from the handling editor and anonymous reviewers are gratefully acknowledged.

\section{References}

[1] M. Borowiecki, On spectrum and per-spectrum of graphs, Publ. Inst. Math. 38 (1985) 31-33.

[2] M. Borowiecki, T. Jóźwiak, On Characteristic and Permanental Polynomials of Multigraphs, Inst. Mat. Fiz., Zielona Góra, 1980, Raport IF-3-80.

[3] M. Borowiecki, T. Jóźwiak, A note on characteristic and permanental polynomials of multigraphs, In: M. Borowiecki, J. W. Kenendy, M. M. Syslo (Eds.), Graph Theory, Lecture Notes in Mathematics: Volume 1018, Springer, Berlin, 1983.

[4] D. M. Cvetković, M. Doob, H. Sachs, Spectra of Graphs, Academic Press, New York, 1980.

[5] Y. Liu, L. You, Further results on the nullity of signed graphs, J. Appl. Math. 2014 (2014) 343-376.

[6] L. Lovász, M. D. Plummer, Matching Theory, Annals of Discrete Mathematics: Volume 29, North-Holland, Amsterdam, 1986.

[7] R. Merris, K. R. Rebman, W. Watkins, Permanental polynomials of graphs, Linear Algebra. Appl. 38 (1981) $273-288$.

[8] H. Minc, Permanents, Addision-Wesly, London, 1978.

[9] L. G. Valiant, The complexity of computing the permanent, Theor. Comput. Sci. 8 (1979) 189-201.

[10] T. Wu, K. C. Das, On the permanental sum of bicyclic graphs, Comput. Appl. Math. 39 (2020) \#72.

[11] T. Wu, H. Lai, On the permanental nullity and matching number of graphs, Linear Multilinear Algebra 66 (2018) $516-524$.

[12] T. Wu, H. Lai, On the permantal sum of graphs, Appl. Math. Comput. 331 (2018) 334-340.

[13] T. Wu, H. Lu, The extremal permanental sum for a quasi-tree graph, Complexity 2019 (2019) \#4387650.

[14] T. Wu, W. So, Unicyclic graphs with second largest and second smallest permanental sums, Appl. Math. Comput. 351 (2019) 168-175.

[15] T. Wu, W. So, Permanental sums of graphs of extreme sizes, Discrete Math. 344 (2021) \#112353.

[16] W. Yan, F. Zhang, On the permanental polynomials of some graphs, J. Math. Chem. 35 (2004) 175-188.

[17] H. Zhang, W. Li, Computing the permanental polynomials of bipartite graphs by pfaffian orientation, Discrete Appl. Math. 160 (2012) $2069-2074$.

[18] H. Zhang, T. Wu, H. Lai, Per-spectral characterizations of some edge-deleted subgraphs of a complete graph, Linear Multilinear Algebra 63 (2014) $397-410$. 